\title{
Facteurs explicatifs de l'adoption des services du mobile money par les clients des opérateurs de téléphonie mobile à Dakar
}

\author{
SOSSOU Maurel Loïs Ahlonko, GAYE Abdoulaye², and WADE Mohamed El Bachir ${ }^{3}$ \\ 1,2 Finance-Organisation-Contrôle-Stratégie, Université Catholique de l'Afrique de l'Ouest, Ziguinchor, Sénégal \\ ${ }^{3}$ Finance-Organisation-Contrôle-Stratégie, Université Cheikh Anta Diop, Dakar, Sénégal
}

\begin{abstract}
Résumé : Le mobile money représente, pour les entreprises de services en l'occurrence les opérateurs de télécommunication, une innovation incrémentale de service accessible à tous les individus via le téléphone portable. Ressortir les facteurs explicatifs de son adoption par les clients répond aux préoccupations des offreurs de services financiers mobiles, en particulier celles des opérateurs de téléphonie mobile qui ambitionnent de développer leur portefeuille clients. Au plan individuel la théorie unifiée d'acceptation et d'utilisation de la technologie a été mobilisée dans différents contextes pour prédire le comportement d'adoption d'une innovation. Grâce à une approche méthodologique mixte et à l'échantillonnage de commodité, une étude qualitative de type semi-structuré suivant une analyse de contenu thématique a été effectuée d'une part et d'autre part un questionnaire conçu à base de Likert à 5 points a été réalisé sur un échantillon de 251 clients utilisateurs. Les résultats révèlent que l'intention des clients à utiliser les services s'explique par la testabilité et l'utilité perçue. Quant à l'analyse de régression logistique, les résultats dévoilent d'une part que l'offre des services du mobile money est à faible coût et d'autre part que l'intention d'utilisation et la commodité constituent les réels facteurs expliquant l'adoption des services du mobile money.
\end{abstract}

Mots-clés : Intention d'utilisation, Adoption, service du mobile money, UTAUT.

\section{INTRODUCTION}

Le progrès rapide de la technologie sans fil et la forte pénétration du téléphone portable ont bouleversé les attitudes et les comportements des individus dans le monde en particulier les pays en développement. Pourtant, une bonne partie de la population n'a pas accès aux services financiers formels en Afrique Subsaharienne, notamment dans l'espace UEMOA. Fortement inspiré des expériences réussies du Kenya avec son M-PESA (M signifie mobile ; PESA fait référence à l'argent en swahili) (Kikulwe et al., 2014), le Sénégal a commencé à utiliser l'argent mobile (mobile money) sous le vocable Orange money depuis 2010. Conscient de la situation depuis 2015, la Banque Centrale des Etats de l'Afrique de l'Ouest (BCEAO) a pris l'initiative de renforcer l'accès aux services financiers des populations vulnérables à travers un support de seconde génération 
communément appelé « mobile money ou argent mobile ». Selon l'Observatoire de la Qualité des Services Financiers (2019), le mobile money désigne l'offre des services financiers permettant aux usagers d'effectuer des activités financières via le téléphone portable.

Faisant partie intégrante de l'écosystème des services financiers mobiles, les opérateurs de téléphonie mobile (OTM) de la plupart des pays en développement en sont à différentes étapes de sa mise en œuvre (Tobbin et Kuwornu, 2011). Du côté de l'offre, au Sénégal, l'expansion de l'argent mobile a été spectaculaire depuis les expériences récentes d'Orange Money, de Free money, de Wizall money, de Wave entre autres.

Depuis quelque temps, l'innovation incrémentale a permis aux entreprises de saisir l'opportunité d'offrir de nouvelles formes de services via le mobile. Pour que ces dernières profitent de l'investissement dans le secteur, elles doivent chercher à comprendre comment les clients réagissent à cette prolifération technologique (Cheikho, 2015). Malgré ses nombreux avantages comme la facilité d'utilisation, l'accessibilité, l'ubiquité, la sécurité, et la compatibilité (Chaix et Torre, 2015), bon nombre d'opérateurs de téléphonie mobile au Sénégal ignorent jusqu'à présent les facteurs qui expliquent l'adoption de cette innovation par les utilisateurs. Ceci dit, chercher à questionner les comportements des consommateurs voire connaitre les facteurs qui expliquent « l'adoption des services » devient nécessaire. Ce concept « adoption » à la fois englobe, l'acceptation, l'essai, l'utilisation et l'internalisation d'une technologie (Lapointe, 1996). Mais cette adoption soulève des problèmes de protection comme la divulgation inappropriée des accusations liée à la falsification des comptes électroniques des consommateurs, la lenteur lors des réclamations, la perturbation du réseau qui entraine un arrêt du système, le manque de liquidité des agents et le coût élevé lié au retrait de fonds (Njoroge, 2016) cité par Baganzi et al. (2017).

Théoriquement, la plupart des travaux s'inscrivent dans une dimension de marketing bancaire (Fall et al., 2019) et plusieurs théories en psycho-sociaux ont été mobilisées pour prédire et expliquer l'acceptation et l'utilisation d'une innovation. Mais la question à se poser est de savoir si les facteurs du modèle UTAUT de Venkatesh (2003, 2012) sont-ils suffisants ou trop pour expliquer l'adoption d'une innovation au plan individuel dans le contexte sénégalais plus précisément à Dakar? En clair, les recherches scientifiques sur le nouveau phénomène consistant à offrir des services financiers à tous en particulier aux personnes non bancarisées (mobile money) sont généralement considérées comme rares (Maurer, 2008 ; Tobbin et Kuwornu, 2011 ; Kikulwe et al., 2014 ; Upadhyay et Jahanyan, 2016). Autrement, étudier le comportement des consommateurs face à l'adoption des services du mobile money dans la ville de Dakar, peut apparaitre intéressant. Ce qui, par conséquent, me permet légitimement de poser la question suivante : quels sont les facteurs explicatifs de l'adoption des services du mobile money par les clients des OTM à Dakar ?

Dans le cadre de cette étude, sont, d'une part mise en évidence, les fondements théoriques et les aspects liés à la méthodologie. D'autre part, les résultats du modèle logistique sont présentés et discutés par rapport au contexte d'étude et à la littérature. 


\section{ETAT DE L’ART ET HYPOTHESES DE RECHERCHE}

Dans cette section, sont passées en revue les études antérieures liées à l'adoption des services bancaires/financiers mobiles et les hypothèses de recherche.

\section{A. Facteurs explicatifs de l'adoption d'une innovation incrémentale de service}

L'adoption d'une innovation par une organisation peut se faire soit par ses employés soit par ses clients, qu'ils soient acquis ou potentiels (Mlaiki, 2012). La littérature associée aux services bancaires mobiles offre des informations précieuses sur l'adoption des services du mobile money, car dans les deux cas, les utilisateurs sont confrontés aux systèmes de paiement électronique, mais il existe certaines différences dans la façon dont les clients perçoivent leur valeur (Schierholz et Laukkanen, 2007 ; Upadhyay et Jahanyan, 2016). Cette littérature a donc pris deux directions générales: La première porte sur l'analyse des facteurs socioéconomiques du grand public dans leur choix d'adopter les services du mobile banking afin d'améliorer leur condition de vie. La deuxième, qui domine les travaux en marketing bancaire, réside dans l'adoption par les consommateurs. Elle met en évidence l'influence des caractéristiques intrinsèques sur l'intention de l'individu à adopter les services du mobile banking (Fall et al., 2019). Mais dans la présente étude, l'analyse est axée sur les facteurs de l'adoption des services du mobile money par les clients abonnés.

Plusieurs chercheurs ont utilisé en psycho-sociologie et en marketing les théories d'acceptation et d'utilisation de l'innovation pour prédire le comportement d'adoption des services du mobile money (Venkatesh et al., 2012 ; Tobbin et Kuwornu, 2011 ; Choudrie et al, 2018). L'élaboration du modèle d'adoption d'une innovation a pour premier repère le développement de la théorie de diffusion de l'innovation (TDI) (Rogers, 1995) qui est un modèle utilisé et développé principalement pour expliquer l'acceptation et l'adoption d'une idée ou d'une pratique perçue comme nouvelle au sein d'un système social. De cette théorie, la communauté scientifique a eu recours à d'autres modèles, notamment la théorie de l'action raisonnée (TAR) de Fishbein et Azjen (1975), qui suggère que le comportement est directement et uniquement déterminé par l'intention comportementale, qui elle-même résulte de deux variables à savoir l'attitude et la norme subjective ; le modèle d'acceptation de la technologie (MAT) de Davis (1989), repose sur le principe que l'utilité perçu et la facilité d'utilisation perçue sont les facteurs fondamentaux de l'utilisation d'un système informatique ; la théorie du comportement planifié (TCP) de Azjen (1991) est une extension de la TAR avec l'ajout du contrôle comportemental perçu, qui est une théorie complète des facteurs les plus proches de la conduite sociale et enfin la théorie unifiée d'acceptation et d'utilisation de la technologie (UTAUT) (Venkatesh \& al. 2003). Par ailleurs, des chercheurs en combinant la théorie de la diffusion de l'innovation de Roger (1995) et le modèle d'acceptation de la technologie (TAM) ont réussi à prédire efficacement le comportement d'adoption d'une innovation (Tobbin et Kuwornu, 2011, Chauhan, 2015). Il est à souligner que la théorie unifiée d'acceptation et d'utilisation de la technologie (UTAUT) pouvait substituer le TAM (Slade et al., 2015), pour d'autre, il était préférable de cumuler des modèles, le cas d'Oliveira et al. (2014) qui, en associant le modèle TAM, UTAUT et modèle de confiance initiale (MCI) ont pu prédire le comportement d'adoption de l'innovation incrémentale de service. 
L'un des modèles le plus appliqué, testé et affiné avec un potentiel significatif pour expliquer l'adoption d'une innovation inversée est UTAUT (Shaikh et Karjaluoto, 2015) qui prend en compte un plus grand nombre de concepts mettant l'accent sur les aspects plus pertinents liés aux consommateurs finaux. L'UTAUT indique qu'il existe trois déterminants directs de l'intention d'utilisation (espérance de performance, espérance d'effort et influence sociale) et deux déterminants directs du comportement d'utilisation (intention comportementale et conditions de facilitation) (Venkatesh et al., 2003). Par ailleurs, l'étude menée par Alkhunaizan (2012), a examiné l'acceptation du commerce mobile en prenant comme outil d'analyse UTAUT amélioré avec les variables confiance et prix perçu. Son modèle révisé a été appliqué à 574 usagers dans plusieurs villes en Arabie Saoudite. Les résultats de cette recherche mettent en évidence que le coût, l'espérance de l'effort et la performance de l'espérance sont autant de facteurs qui ont un effet sur l'intention d'utiliser le commerce mobile. Dans la même dynamique Martins et al. (2014) se sont appuyés sur la théorie UTAUT avec l'ajout du risque perçu pour analyser l'intention comportementale de l'utilisation des services bancaires par l'Internet en Portugal. Leur modèle confirme 52\% de la variance de l'intention comportementale et son pouvoir explicatif croit à 56\% prenant en compte les variables modératrices (l'âge et le sexe). De même, Lafraxo et al. (2018) ont mobilisé UTAUT avec l'ajout de la confiance, la sécurité et du risque perçu pour expliquer l'adoption des services du mobile banking au Maroc. Les résultats indiquent que l'effort attendu, la performance espérée, l'influence sociale et la sécurité ont un impact significatif sur l'intention comportementale.

Cet article s'inscrit dans le cadre de certains travaux (Venkatesh et al., 2003, 2012 ; Min et al., 2008 ; Lafraxo et $a l ., 2018$; Mensah et $a l ., 2020$ ) et vise à contribuer au développement théorique de l'identification des facteurs de l'adoption des services du mobile money par les clients des OTM dans la ville de Dakar.

\section{B. Hypothèses de recherche}

Avant de formuler les hypothèses de recherche dans cette section, il a été question de mener une étude qualitative dont les résultats sont présentés ci-dessous.

\section{- Démarche qualitative exploratoire}

Les résultats sont présentés en fonction de 2 grandes catégories retenues (facteurs de résistance et facteurs de motivation) sous forme de tableaux en vue d'être interprétés.

Lors du codage, nous avons retenu les passages du verbatim répondant aux caractéristiques de facteurs de motivation ou de l'adoption de façon explicite comme unité d'enregistrement. Il s'agit des passages qui font ressortir des raisons pertinentes qui peuvent freiner ou motiver l'adoption des services d'argent mobile. Par ailleurs, les réponses ou raisons qui, de façon évidente ou triviale, renvoient aux facteurs d'adoption par les interviewés telles que disposer d'un revenu, d'un téléphone portable, d'un salaire, etc. n'ont pas été pris en compte. En outre, les réponses traduisant une indécision de la part de l'interviewé ont été pris en compte suivant leur occurrence pour justifier leur regroupement comme facteur de motivation ou de résistance. Les avis ne collant pas à la définition de facteurs de motivation ou de résistance n'ont pas été pris en compte. De 
même que les facteurs propres à chaque individu tels la pauvreté, l'analphabétisme, le manque d'instruction ou d'éducation. Les variables prenant une valeur de variables post-adoption c'est-à-dire traduisant la poursuite d'utilisation pour aboutir à une relation de fidélité après adoption des services n'ont pas non plus été pris en compte. L'adoption étant perçue comme le processus au cours duquel le consommateur se considère comme client abonné qui utilise de façon régulière les services de mobile money.

Nous avons, par ailleurs opté pour une approche descriptive visant à faire ressortir les facteurs de motivation à l'adoption des services du mobile money. En effet, en ce qui concerne la réduction de l'analyse de contenu au seul « contenu manifeste », De Bonville et Guerard (2000) indique qu'il n'y a pas lieu de considérer comme inutile et invalide toute démarche descriptive limitée au seul contenu manifeste des messages. Il ajoute que même si elles ne proposent aucune inférence, bon nombre d'analyses de contenu conservent leur utilité puisqu'elles extraient des messages des caractères autrement demeurés imperceptibles ou confus. Quant à l'unité d'énumération, c'est-à-dire l'unité qui enregistre les occurrences des unités d'enregistrement, nous avons dénombré toutes les fréquences d'apparition desdites unités d'enregistrement indépendamment du nombre d'interrogés.

Rappelons que cette étude qualitative s'inscrit dans une logique compréhensible en privilégiant la description des processus plutôt que l'explication des causes. Alors l'analyse de contenu thématique sera mobilisée afin de ressortir si possible d'autres facteurs spécifiques au contexte de l'étude.

$\mathrm{Au}$ total 20 entretiens semi-directifs ont été effectués durant le mois de février 2019. La récolte des informations auprès des clients abonnés, d'une durée d'environ 45 minutes $^{1}$, a été réalisée suivant plusieurs modes d'administration (face à face, par visio-conférence, et par WhatsApp). Les informations les concernant sont renseignées dans le tableau $\mathrm{n}^{\circ} 1$.

Tableau n ${ }^{\circ} 1$ : Caractéristiques démographiques des interviewés

\begin{tabular}{|l|c|}
\hline Genre & 12 \\
Hommes & 8 \\
Femmes & \\
Age & 11 \\
$25-40$ ans & 9 \\
$41-52$ ans & 5 \\
Situation matrimoniale & 15 \\
Célibataire & \\
Marié (e) & 2 \\
Niveau d'étude & 6 \\
Analphabète & 12 \\
Secondaire & \\
Universitaire & 14 \\
Revenu & 4 \\
$137 €-412 €$ & 2 \\
$412 €-686 €$ & \\
$+686 €$ & \\
\hline
\end{tabular}

${ }^{1}$ Cette durée correspond à la durée moyenne des entretiens semi-directifs généralement réalisés dans les travaux de recherches en marketing (Evrard et al., 2003). 
Ayant pour but de comprendre les réels facteurs expliquant l'adoption ou non des services d'argent mobile à Dakar par les interviewés, le guide d'entretien a été élaboré autour de deux thèmes : l'expérience et la fréquence d'utilisation des services d'argent mobile, les fondamentaux qui empêchent ou qui motivent l'adoption des services du mobile money. Les données collectées ont été retranscrites sous forme de verbatim et sont ensuite passées à la phase de codage formant notre corpus (Annexe : A).

Les résultats issus de l'étude qualitative exploratoire nous poussent à avoir un modèle adapté composé de neuf (08) variables prenant en compte les spécificités du contexte de l'étude (Voir Tableau n²). Bien que surprenante, ces facteurs correspondent aux concepts existants dans la littérature sans oublier une nouvelle variable «testabilité » décelée lors des entretiens semi-structurés.

Tableau 2 : Facteurs de motivation et de résistance en fonction de leurs occurrences

\begin{tabular}{|c|c|c|}
\hline Catégories & Unités de sens & Occurrences \\
\hline \multirow{7}{*}{$\begin{array}{lll}\text { Facteurs } & & \text { de } \\
\text { motivation et } & \text { de } \\
\text { résistance } & & \end{array}$} & facilité d'utilisation & 8 \\
\hline & commodité du service & 28 \\
\hline & testabilité du service & 19 \\
\hline & influence sociale & 14 \\
\hline & utilité perçue & 17 \\
\hline & coût du service & 7 \\
\hline & $\begin{array}{c}\text { risque perçu (risque financier et risque de } \\
\text { confidentialité) }\end{array}$ & 7 \\
\hline
\end{tabular}

Ici le focus sera mis sur la variable « testabilité » décelée lors de l'étude qualitative exploratoire qui révèle et confirme la présence de (08) variables issues des théories existantes et des travaux empiriques. La raison la plus simple est que la plupart des modèles théoriques privilégiés sont issus des travaux africains (Venkatesh et al., 2003, 2012 ; Tobbin et Kuwornu, 2011 ; Lafraxo et al., 2018 ; Mensah et al., 2020).

\section{- Testabilité de l'innovation incrémentale}

La possibilité d'essai des services par les clients abonnés a aussi été énumérée comme cause de motivation à l'adoption des services du mobile money. Comme raison, ils avancent que «La phase d'essai est primordiale dans l'adoption de tout produit/service»(A.S, 27 ans, M) ${ }^{2}$, ou encore que «Le produit me semble plus pratique après l'avoir testé » (M.S, 31 ans, F). Sans code secret, d'autres interviewés trouvent que sans cette phase d'essai, qui permet en même temps d'évaluer les performances de l'innovation, on ne peut décider si l'on doit adopter ou pas les services qu'offre cette innovation et affirment que «le mobile money reste une innovation et perturbe forcément les habitudes et donc tout service ou produit aura une phase de test avant d'être adopté » (A.V, 35 ans, M ; M.V, 28 ans, F). Ainsi, dans un souci de parcimonie et pour simplifier le modèle de recherche, l'influence des variables modératrices de l'UTAUT ne sera pas retenue dans le test. Voici les hypothèses émises.

${ }^{2}$ Initiale de la personne interviewée, son âge et son sexe 


\section{- L'intention d'utilisation et les variables fonctionnelles}

L'intention d'utilisation dépend des variables fonctionnelles (H1). Parmi les variables fonctionnelles susceptibles d'influencer l'intention d'utiliser les services du mobile money par les clients, nous avons retenu pour cette étude : l'effort attendu, le risque perçu et la testabilité.

- $\quad$ L'effort attendu

Il représente le degré de facilité associé à l'utilisation d'un système (Venkatesh et al., 2003). Autrement, il définit la simplicité des procédures pour remplir une exigence de service avec l'outil technologique (BuabengAndoh, 2012). De plus, l'effort attendu reflète la qualité du support utilisateur et la facilité d'utilisation des étapes de transaction (Lubua et al., 2017). Dans leurs recherches sur l'adoption du mobile money, certains auteurs ont montré que l'effort attendu influence de manière positive l'intention d'utilisation des services d'argent mobile (Kikulwe et al. 2015 ; Lubua et al. 2017).

A partir de ce qui précède on peut émettre l'hypothèse suivante :

Hypothèse 1a : l'effort attendu a une influence positive et significative sur l'intention d'utilisation des services du mobile money.

- Le risque perçu

Dans les travaux portant sur l'adoption des services du mobile money, il représente pour les consommateurs un obstacle important (Tobbin et Kuwornu, 2011). Le même auteur définit le risque perçu comme la conviction du consommateur quant aux conséquences négatives potentielles incertaines de la transaction d'argent mobile. Pour Bauer (1967) le risque perçu est défini comme une combinaison d'incertitude et de gravité des résultats impliqués c'est-à-dire qui s'appréhende comme la conviction que des événements inattendus peuvent se produire. Ainsi, il a été constaté que la réduction de l'incertitude a une influence positive sur l'intention des consommateurs d'adopter des systèmes transactionnels électroniques (Chen, 2008). C'est la raison pour laquelle l'effet du risque perçu, en tant que construction singulière, sur l'intention comportementale a été à la fois soutenu dans certaines études (Lu et al., 2011 ; Yang et al., 2012), et rejeté dans d'autres (Tan et al., 2014), dans le contexte du paiement mobile. Il est aussi possible que l'utilisateur d'argent mobile subisse des pertes financières ou des informations personnelles du fait de l'utilisation de transactions mobiles (Aljabri, 2015). Le risque perçu lié à l'utilisation de l'argent mobile est supposé être plus important que celui lié à l'utilisation des services bancaires par Internet (Nel et al., 2014). Lafraxo et al. (2018) ont exploité une version étendue de l'UTAUT intégrant le risque perçu dans leur étude sur les facteurs d'adoption des applications bancaires mobiles au Maroc. L'analyse des 460 réponses obtenues a montré que le risque perçu dans l'application mobile influence négativement l'intention comportementale des usagers.

Dans ce papier, le risque perçu prend en compte deux dimensions à savoir : le risque de confidentialité (risque lié à une perte potentielle de contrôle des informations personnelles qui seraient divulguées lors de l'utilisation de l'innovation) et le risque financier (correspond aux pertes financières dues à la fraude dans le contexte 
élargit des services financiers). Ainsi, plus le risqué est élevé, plus les clients auront moins l'intention d'utiliser la technologie mobile. Le signe espéré est négatif.

Hypothèse $\mathbf{1 b}$ : le risque perçu influence négativement et significativement l'intention d'utiliser les services du mobile money.

- La testabilité

Elle est appréhendée comme le degré auquel une innovation peut être expérimentée sur une base limitée avant de prendre une décision d'adoption (ou de rejet) (Agarwal et Prasad, 1997). Dans le but de faire connaitre une innovation voire un produit nouveau ou service aux clients potentiels, il serait judicieux pour une organisation d'offrir à ses derniers la possibilité d'expérimenter l'innovation afin de les inciter à prendre l'engagement de l'utiliser (Agarwal et Prasad, 1997). Ainsi, l'adoption du transfert d'argent mobile est plus probable si la technologie est démontrée à l'utilisateur ou si elle peut être utilisée gratuitement lors de la première utilisation. Des recherches antérieures soutiennent que les anciens utilisateurs d'une innovation perçoivent la testabilité comme plus importante que les utilisateurs ultérieurs (Tobbin et Kuwornu, 2011). Après avoir mené une étude sur l'adoption du transfert d'argent mobile au Ghana auprès de 298 répondants, ils conclurent que la testabilité affecte de manière significative l'intention d'utiliser le transfert d'argent mobile, poursuit les mêmes auteurs. Ainsi, l'intention d'utiliser est plus probable si cette innovation est démontrée à l'utilisateur ou s'il trouve l'occasion de la tester lors de sa première utilisation.

Hypothèse 1c : la testabilité a une influence positive et significative sur l'intention d'utilisation des services du mobile money.

\section{- L'intention d'utilisation et les variables extra-fonctionnelles}

L'intention d'utiliser les services du mobile money dépend de l'avantage des variables extra-fonctionnelles (H2). Parmi les facteurs extra-fonctionnels susceptibles d'influencer l'intention d'utilisation des consommateurs, nous avons : la performance espérée et l'influence sociale.

- Utilité perçue

La performance espérée est remplacée par l'utilité perçue, car elle convient plutôt pour évaluer l'efficacité au travail au niveau entreprise mais pas au niveau individuel (Min et al., 2008). Etant donné que les consommateurs représentent l'échantillon de cette étude, nous avons pris en compte cette modification dans ce papier. Donc l'utilité perçue représente le degré auquel un individu croit que l'utilisation d'un système l'aidera à obtenir des gains de performance au travail (Venkatesh et al. 2003). De même, elle est simplement le niveau auquel l'utilisateur perçoit que les outils sont suffisamment bons pour bénéficier de ses activités (Aboelmaged et Gebba, 2013). D'après Rogers (1995) il suggère dans son étude que pour qu'une nouvelle technologie se diffuse rapidement dans la communauté, les utilisateurs doivent avoir une raison claire pour son adoption. Cette assertion montre que l'augmentation de l'utilité perçue influence l'intention d'utilisation. Également, Venkatesh et al. (2003) conclurent que la variable est essentielle pour déterminer l'intention de 
l'utilisateur à adopter une nouvelle technologie. L'auto-efficacité, l'accessibilité de la technologie, les caractéristiques du système représentent les raisons de l'utilité perçue de la nouvelle technologie (Hsieh et al., 2017). Les auteurs (Baptista et Oliveira, 2016; Basri, 2018) ont montré de manière empirique que les utilisateurs de services bancaires mobiles pensent que l'utilité perçue est l'un des antécédents les plus importants de l'intention comportementale. On pourrait dire que plus l'individu s'aperçoit que l'utilisation d'une technologie lui procure un avantage relatif, plus il aura l'intention de l'utiliser. A cet égard, l'hypothèse suivante est émise :

Hypothèse 2a: L'utilité perçue a une influence positive et significative sur l'intention d'utilisation des services du mobile money.

- Influence sociale

Encore appelé « normes sociales » ou «normes subjectives », elle est définie comme le degré de perception par un individu que d'autres personnes importantes pour lui pensent qu'il devrait utiliser le nouveau système (Fishbein et Ajzen, 1975 ; Venkatesh et al., 2003). D'après Martins et al. (2014), l'influence sociale affecte l'intention des utilisateurs à adopter les services bancaires par Internet. Khatimah et al. (2019) ont réalisé une enquête auprès de 249 utilisateurs de monnaie électronique en Indonésie. Les résultats empiriques ont révélé que la motivation hédonique et l'influence sociale avaient un impact significatif sur les habitudes de paiement. En réalité, on retient que plus l'utilisateur est influencé par les personnes qui lui sont proches (Parents, amis,), plus il aura l'intention d'utiliser la nouvelle technologie. Le signe espéré est positif.

Hypothèse $2 \boldsymbol{b}$ : l'influence sociale a un effet positif et significatif sur l'intention d'utiliser

- Les conditions facilitantes et l'intention d'utilisation et l'adoption des services du mobile money L'adoption de l'innovation incrémentale de service est influencée par les conditions facilitantes (H3). Par ailleurs, les conditions facilitantes ont été remplacées par la commodité et le coût dans une étude menée en Chine (Min et $a l .$, 2008). Leur étude a mobilisé le modèle UTAUT, prenant en compte ce changement pour étudier l'acceptation et l'utilisation du m-commerce. Cette modification est prise en compte dans ce papier.

- La commodité

Elle est définie comme « les dépenses perçues de temps et d'efforts par les consommateurs pour affecter une transaction » (Berry et al., 2002). Selon Brown (1990), la commodité prend en compte cinq dimensions, à savoir : le temps, le lieu, l'acquisition, l'utilisation et l'exécution. En clair, cette variable offre plus l'ubiquité aux utilisateurs. Lichtenstein et Williamson (2006) indiquent que le gain de temps et l'accès $24 \mathrm{~h} / 24$ et $7 \mathrm{j} / 7$ semblent être les aspects les plus importants de la commodité des services bancaires par Internet. L'une des études portant sur l'adoption du mobile banking a révélé que la relation la plus significative était entre la commodité et l'adoption d'une technologie (Bhatiasevi, 2016). Ainsi donc, plus les services du mobile money procurent un niveau de commodité élevé, plus les consommateurs vont l'adopter. Le signe espéré est positif. Hypothèse $3 a$ : La commodité a une influence positive et significative sur l'adoption des services du mobile money. 
SOSSOU et al.: Facteurs explicatifs de l'adoption des services du mobile money par les clients des opérateurs...

- $\quad$ Le coût perçu

Encore appelé coût financier perçu, est défini comme « la mesure dans laquelle une personne pense que l'utilisation des services bancaires mobiles coûtera de l'argent » (Luarn et Lin, 2005). Également, c'est le compromis cognitif des consommateurs entre les avantages perçus de l'utilisation de la technologie et de son coût monétaire qu'elle engendre (Venkatesh et al., 2012). Siddik et al. (2014) postule que les coûts de transaction tels que les frais de procédures, de retrait et de dépôt ont une influence sur l'intention comportementale des consommateurs d'adopter des services de transfert d'argent mobile. Ce qui peut influencer la décision d'utiliser ou non les services. Pour Hamza (2014) les facteurs socio-économiques et les coûts de transaction sont également conçus pour avoir des liens étroits avec l'intention comportementale d'utiliser l'argent mobile. De plus, Mohamad et al. (2019) ont montré dans leur étude que le coût financier à une relation significative avec l'adoption du paiement électronique en Malaisie. On peut donc retenir que plus les services coûtent cher par rapport aux autres canaux électroniques plus les clients hésiteront à l'adopter de par leur comportement. Le signe espéré est négatif.

Hypothèse $3 \boldsymbol{b}$ : le coût perçu a un effet négatif et significatif sur l'adoption des services du mobile money.

\section{- L'intention d'utilisation et l'adoption des services du mobile money}

- L'intention

Elle est une composante conative intermédiaire entre l'attitude et le comportement (Fishbein et Ajzen, 1975).

L'intention est souvent le fait, de se proposer un but, de poser un acte voire un dessein avec un objectif donné.

Elle engendre des idées comme « je dois faire », « je vais faire » ou encore « je le ferai » (Limayem et Rowe, 2006). De nombreuses études supposent et confirment que l'intention est le facteur qui prédit et qui représente le meilleur pronostic du comportement d'un consommateur (Fishbein et Ajzen, 1975 ; Martins et al., 2014). Parce qu'elle permet de manière indépendante à l'individu d'intégrer des informations pertinentes qui peuvent impacter son comportement réel. Autrement, une intention positive d'utiliser une technologie influence forcément l'utilisation réelle de cette dernière (Venkatesh et al., 2003). Des études en mobile banking ont montré l'influence positive de l'intention d'utilisation sur l'utilisation actuelle (Yu, 2012). Cette assertion nous pousse à émettre l'hypothèse suivante :

\section{Hypothèse 4 : L'intention d'utilisation a une influence positive et significative sur l'adoption des services} du mobile money. 


\section{FIGURE I : MODELE CONCEPTUEL DE L'ETUDE}

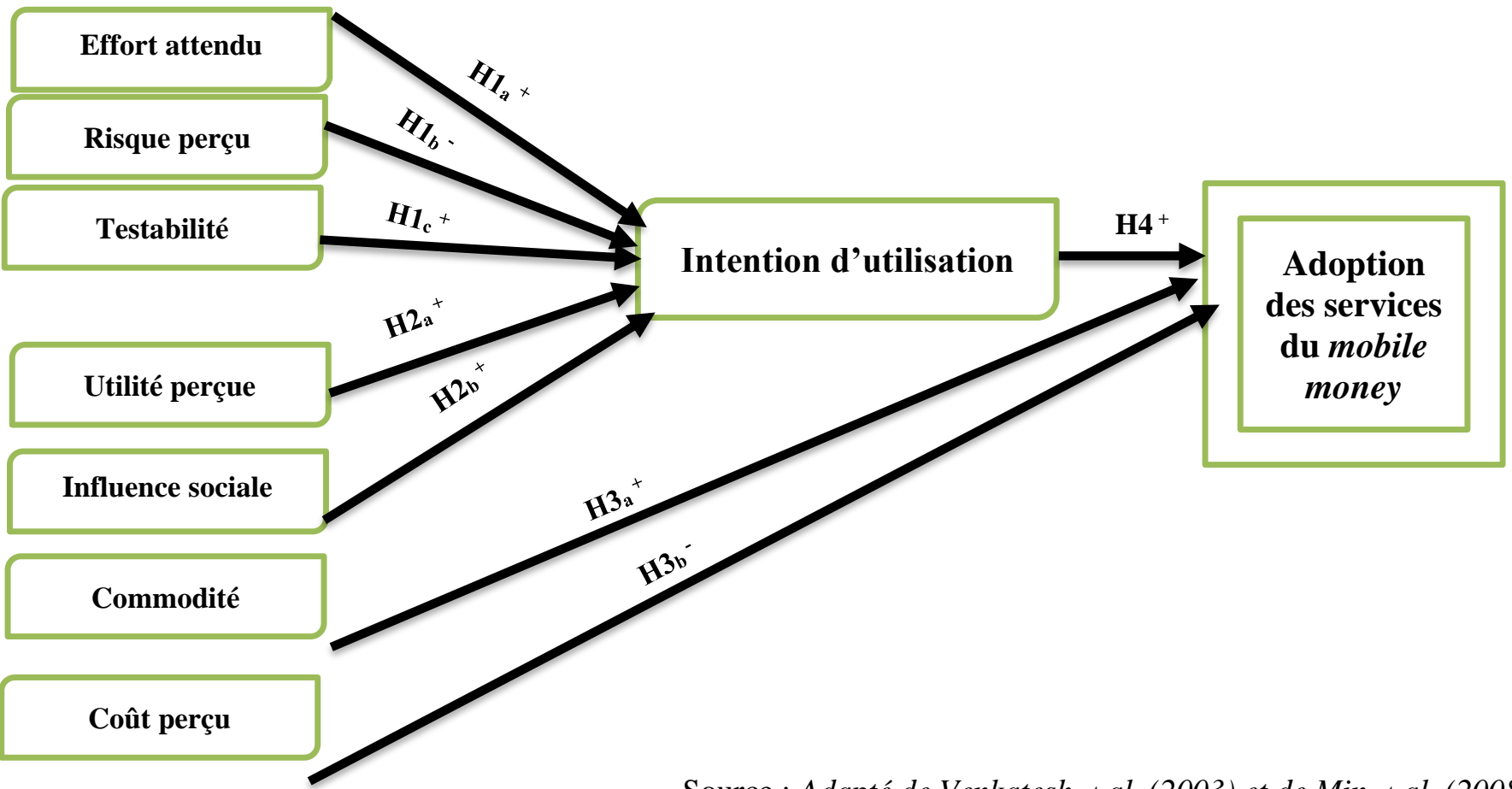

Source : Adapté de Venkatesh et al. (2003) et de Min et al. (2008)

\section{APPROCHE METHODOLOGIQUE}

Le positionnement épistémologique de l'étude s'inscrit dans un courant positiviste suivant une logique hypothético-déductive. Il a été souligné que la méthodologie ne représente qu'un aspect de l'épistémologie (Jolibert et $a l .$, 2012). L'étude simultanée par démarches qualitative et quantitative a été effectué. L'étude qualitative expliquée ci-dessus avec la littérature a permis de déceler la variable « testabilité » pris en compte dans le modèle de recherche. Dans cette section, les options méthodologiques relatives au recueil de données, aux méthodes d'analyse de données et à la mise en œuvre d'instruments de mesure seront mises en évidence et justifiés.

\section{A. Récolte de données et description de l'échantillon}

Pour assurer l'indépendance entre le sujet et l'objet d'étude nous avons opté pour une approche quantitative. Les recherches de nature quantitative favorisent le questionnaire comme un instrument de recueil de données (Thietart, 2014). De novembre en décembre 2019, nous avons réalisé une enquête par questionnaire en face à face auprès des clients utilisateurs des services du mobile money dans la zone urbaine en région Dakaroise (Parcelles assainies, Dakar-Plateau, Grand-Dakar, HLM Grand-Yoff, Médina, Liberté 4, Liberté 6, Almadies, Ouakam). Sur les 300 consommateurs ciblés, 251 ont participé à l'enquête accumulant un taux de réponse de 83\%. La répartition par genre des sujets de l'étude était respectivement de 59,3\% d'hommes et 40,7\% de femmes. Les utilisateurs âgés de 25 à 40 ans formaient un groupe d'âge le plus important (59,7\%), suivis des utilisateurs jeunes ayant au plus 24ans $(23,1 \%)$ et enfin des répondants âgés au-delà de 41 ans $(17,2 \%)$. Si $62,6 \%$ d'entre eux ont un revenu entre 180000 et 450000 FCFA voire plus, il faut souligner que plus de 37,4\% ne disposent pas de revenu supérieur à 90000 FCFA. Ils étaient soit des salariés (38,3\%), des entrepreneurs 
SOSSOU et al.: Facteurs explicatifs de l'adoption des services du mobile money par les clients des opérateurs...

(28,2\%), des étudiants $(25,1 \%)$ ou encore des chômeurs (8,4\%). Par ailleurs, 33,4\% des utilisateurs n'avaient pas le Baccalauréat (BAC) voire aucun diplôme et 66,6\% étaient des instruits (BAC+2 et plus). Au final 47,4\% étaient mariés contre 52,6\% célibataires. Tout ceci grâce à un échantillonnage par convenance.

\section{B. Méthode d'analyse des données}

Les données collectées sont traitées à l'aide du logiciel SPSS. Les principales analyses ont permis de réaliser des tests, de fiabilité, de validité, et de régression linéaire. Par la suite, la variable « adoption des services du mobile money» avait été mesurée avec une échelle de Likert à 05 points. Aux fins d'une analyse plus approfondie, cette variable a été codée en tant que variable binaire (avec $1=\mathrm{j}$ 'utilise actuellement et $0=$ jamais utilisé), pour effectuer une régression logistique. Cette méthode est très appropriée pour expliquer l'effet d'une ou plusieurs variables explicatives sur une variable à expliquer mesurée sur une échelle booléenne » (Jolibert et $a l ., 2012$ ). Dans ce papier, la variable dépendante nominale «Adoption des services du mobile money » est expliquée par les variables explicatives métriques « intention d'utilisation», «la commodité » et «le coût perçu».

\section{Instruments de mesure des variables indépendantes}

Du point de vue méthodologique, nous avons mobilisé le modèle UTAUT adapté de Venkatesh et al. (2003, 2012) et Min et al. (2008) avec l'ajout de la testabilité décelée dans l'étude qualitative et le risque perçu (Tobbin et Kuwornu, 2011 ; Lafraxo et al., 2018). Les éléments de l'enquête ont été développés en adaptant les mesures existantes validées par d'autres chercheurs dans les domaines de la banque mobile et du paiement mobile. Au total 08 construits centrales de l'UTAUT (effort attendu, risque perçu, testabilité, utilité perçue, influence sociale, commodité, coût perçu et intention comportementale), à l'exception de l'adoption de l'innovation, ont été mesurés en utilisant une approche d'échelle de Likert à cinq (05) points allant de 1 (entièrement en désaccord) à 5 (entièrement d'accord).

Voici en résumé l'adaptation des échelles de mesure de chaque variable indépendante en tableaux n³.

Tableau $\mathbf{n}^{\circ} 3$ : Items des variables du modèle de recherche

$\begin{array}{lll}\text { Construits Items } & \text { Source }\end{array}$

Effort Attendu EE1 : Apprendre à utiliser les services du mobile money est facile pour moi.

EE2 : Mon interaction avec le mobile money est claire et compréhensible. Venkatesh et al. (2012)

EE3 : Je trouve le mobile money facile à utiliser

EE4 : Il m'est facile de devenir habile à utiliser le mobile money.

Risque perçu $\quad$ RP1 : Je risque de perdre ma somme quand je paye une facture

d'eau et d'électricité avec soit (Orange money, Free money, E-money)

RP2 : Les informations concernant mes transactions avec

les services du mobile money sont falsifiées.

Featherman et Pavlou (2003)

RP3 : La perturbation du réseau entraine des coûts supplémentaires lors des transactions

RP4 : Pendant l'utilisation du mobile money, les intrus peuvent utiliser

mon compte en ayant accès aux informations.

Ozdemir et Trott (2009) 


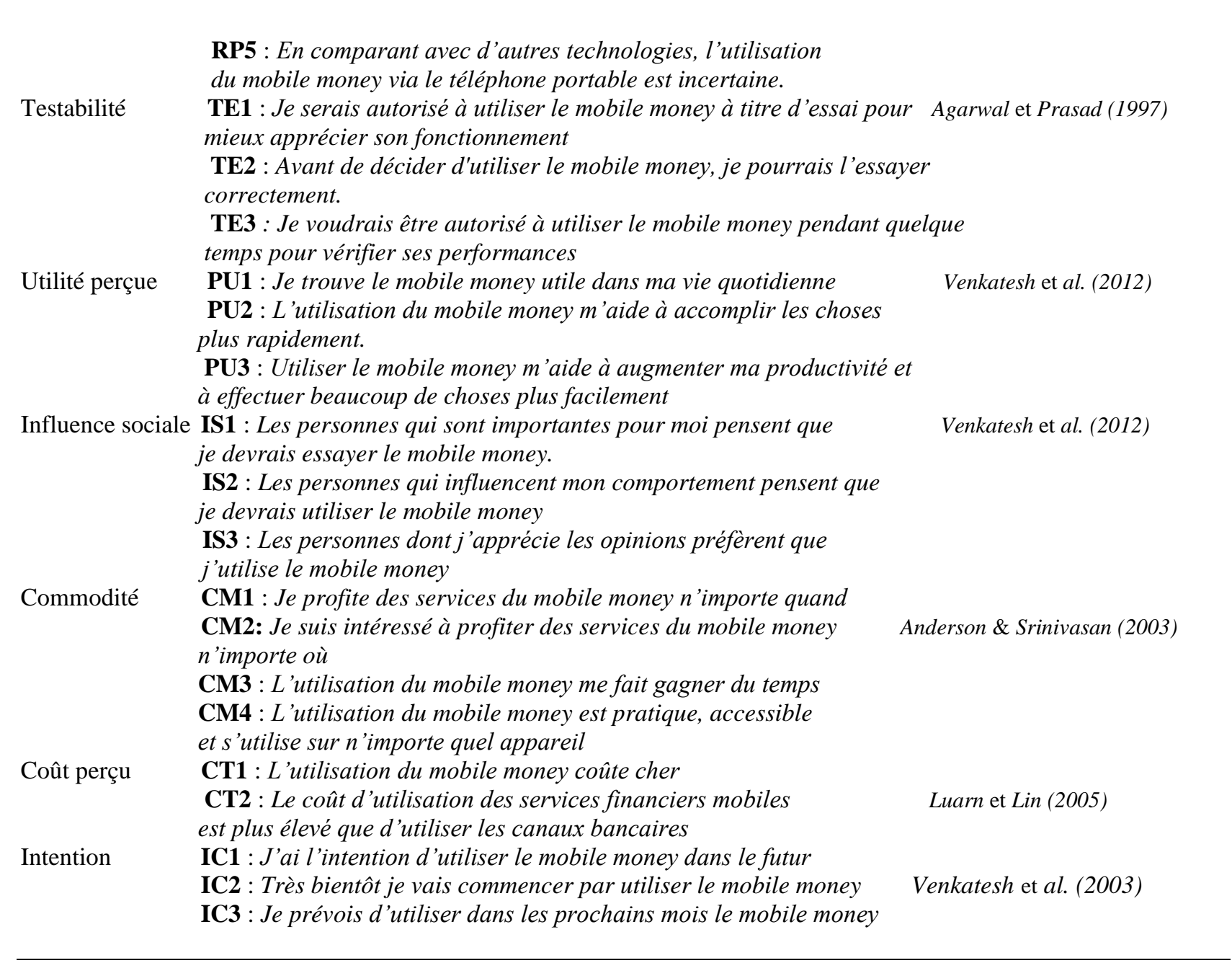

\section{RESULTATS ET DISCUSSIONS}

Dans cette section, sont d'abord effectués les tests de corrélation interne entre les items de chaque échelle, ensuite il sera question de mettre en exécution l'analyse factorielle en composantes principales (ACP) en espérant avoir plus d'un axe factoriel pour pouvoir effectuer une deuxième analyse ACP avec rotation Varimax. Après résultat, il sera question de procéder à l'analyse de régressions. Le tableau ${ }^{\circ} 4$ résume les tests de fiabilité et de validité des échelles de mesure.

Tableau $n^{\circ} 4$ : Analyse factorielle et de fiabilité des variables indépendantes

\begin{tabular}{|l|l|l|l|l|l|l|}
\hline \multicolumn{1}{|c|}{ Facteurs retenus } & $\begin{array}{c}\text { Indice } \\
\text { KMO }\end{array}$ & $\begin{array}{c}\text { Test de } \\
\text { Bartlett }\end{array}$ & $\begin{array}{c}\text { Valeur } \\
\text { propres }\end{array}$ & $\begin{array}{c}\% \\
\text { Variance } \\
\text { expliquée }\end{array}$ & $\begin{array}{c}\text { Alpha } \\
\text { Cronbach }\end{array}$ & $\begin{array}{c}\text { Qualités de } \\
\text { représentations } \\
\text { des Items }\end{array}$ \\
\hline FACT_UTILITE (Trois items) & $\mathbf{0 , 7 4 7}$ & 0,000 & $\mathbf{2 , 8 4 8}$ & 94,933 & $\mathbf{0 , 9 7 3}$ & $>0,5$ \\
\hline FACT_EFFORT (Quatre items) & $\mathbf{0 , 8 8 0}$ & 0,000 & $\mathbf{3 , 7 5 1}$ & 93,786 & $\mathbf{0 , 9 7 8}$ & $>0,5$ \\
\hline FACT_RP (Cinq items) & $\mathbf{0 , 7 4 7}$ & 0,000 & $\mathbf{3 , 4 1 7}$ & 68,342 & $\mathbf{0 , 8 7 7}$ & $>0,5$ \\
\hline FACT_TE (Trois items) & $\mathbf{0 , 7 3 5}$ & 0,000 & $\mathbf{2 , 9 0 5}$ & 96,823 & $\mathbf{0 , 9 8 4}$ & $>0,5$ \\
\hline FACT_CT (Deux items) & $\mathbf{0 , 5}$ & 0,000 & $\mathbf{1 , 7 2 5}$ & 86,241 & $\mathbf{0 , 8 4 0}$ & $>0,5$ \\
\hline FACT_ISA (Trois items) & $\mathbf{0 , 6 9 0}$ & 0,000 & $\mathbf{2 , 5 8 8}$ & 86,282 & $\mathbf{0 , 9 2 0}$ & $>0,5$ \\
\hline
\end{tabular}


SOSSOU et al.: Facteurs explicatifs de l'adoption des services du mobile money par les clients des opérateurs...

\begin{tabular}{|l|l|l|l|l|l|l|}
\hline FACT_COM (Quatre items) & $\mathbf{0 , 8 5 7}$ & 0,000 & $\mathbf{3 , 7 8 7}$ & 94,686 & $\mathbf{0 , 9 8 1}$ & $>0,5$ \\
\hline FACT_IT (Trois items) & $\mathbf{0 , 7 4 5}$ & 0,000 & $\mathbf{2 , 9 6 6}$ & 98,866 & $\mathbf{0 , 9 9 4}$ & $>0,5$ \\
\hline
\end{tabular}

Le seuil d'acceptabilité de l'alpha de Cronbach est en général de 0,7 (Nunnally, 1978). Les variables indépendantes sont mesurées à partir des variables manifestes. Les corrélations entre ces items sont toutes positives et significatives puisqu'elles sont assurées par des alphas de Cronbach acceptables et très bon supérieur à 0,7 . Ces résultats montrent donc qu'il y a une cohérence entre les réponses fournies par les consommateurs des services financiers mobiles.

La valeur du KMO est d'autant meilleure qu'elle est proche de 1 et jugé inacceptable quand elle est inférieure à 0,5 (Evrard et al., 2009; Gavard-Perret et al., 2012). Ceci dit, les indices KMO de toutes les variables indépendantes sont acceptables et leur test de Bartlett est significatif et permet de valider la conduite de chaque analyse factorielle. De cette analyse en composantes principales exploratoires ressort pour chaque variable indépendante un seul axe factoriel dans lequel les variables manifestes expliquent un très bon pourcentage de la variance dont le plus petit se chiffre à $68,342 \%$ et ayant tous une valeur propre supérieure à 1 . Les qualités de la représentation sont supérieures à 0,5 pour tous les items des variables latentes.

\section{A. LES RESULTATS ISSUS DE LA REGRESSION LOGISTIQUE BINAIRE}

Le test de l'UTAUT dans l'environnement des services financiers mobiles s'est concentré d'une part sur l'examen des facteurs influençant l'intention d'utiliser et d'autre part sur les facteurs affectant l'adoption des services du mobile money. Dans un premier temps, les cinq (05) variables indépendantes et l'intention d'utiliser sont étudiées. Par la suite, sont mises en évidence, les résultats issus de la régression logistique. Les résultats obtenus par l'analyse de régression linéaire sont présentés dans le tableau ci-dessous :

Tableau n ${ }^{\circ} 5$ : Résultat de la régression linéaire des variables indépendantes sur l'intention d'utiliser (FACT_IT).

\begin{tabular}{lcccc}
\hline Variables & Coefficients & Erreur Standard & Test t & P-value \\
\hline FACT_UTILITE & 0,453 & 0,044 & 10,284 & 0,000 \\
FACT_EFFORT & $-0,093$ & 0,041 & $-2,287$ & 0,023 \\
FACT_RP & $-0,067$ & 0,029 & $-2,306$ & 0,022 \\
FACT_ISA & 0,050 & 0,034 & 1,478 & 0,141 \\
FACT_TE & 0,607 & 0,035 & 17,196 & 0,000 \\
\hline
\end{tabular}

$$
\mathbf{R}^{\mathbf{2}}=\mathbf{0 , 8 1 5} \quad \text { P-value }=0.000<0,05
$$

La mesure du $\mathrm{R}^{2}$ ajusté montre que $81,5 \%$ de l'intention d'utiliser est expliquée par l'utilité perçue, la testabilité, et le risque perçu.

La relation entre l'effort attendu et l'intention d'utilisation est positive et significative, ce qui est similaire pour l'utilité perçue. Par ailleurs, l'influence de l'effort attendu est significative quand on inclut la variable commodité. Ceci dit quand l'on introduit tous les construits mobilisés dans le modèle, l'influence de l'effort 
attendu sur l'intention d'utiliser les services du mobile money n'est pas maintenue. Par conséquent l'hypothèse $\mathbf{H 1}_{\mathbf{a}}{ }^{+}$est réfutée. Pour la testabilité, c'est-à-dire avoir la possibilité que les fournisseurs qui proposent les services financiers mobiles puissent permettre aux abonnés de l'utiliser à titre d'essai, a un effet positif et significatif sur l'intention d'utilisation. Par conséquent, les résultats soutiennent l'hypothèse $\mathbf{H 1} \mathbf{c}^{+}(\mathbf{l a}$ testabilité a une influence positive et significative sur l'intention d'utiliser les services du mobile money). Les résultats révèlent qu'il y a un lien entre le risque perçu et l'intention d'utiliser. Ce résultat indique que plus l'utilisation des services du mobile money est perçue comme risqué par les clients abonnés, plus ils n'auront l'intention de l'utiliser. Par conséquent l'hypothèse $\mathbf{H 1} \mathbf{1}_{\mathbf{b}}$ ' est non réfutée.

Les variables fonctionnelles influencent l'intention d'utiliser notamment le risque perçu qui influe négativement sur l'intention d'utilisation et la testabilité qui influe positivement et significativement sur l'intention d'utiliser. De ce fait, l'hypothèse 1 n'est pas réfutée.

L'utilité perçue, c'est-à-dire les avantages escomptés obtenus en utilisant les services de l'innovation incrémentale, a un effet positif direct et significatif sur l'intention d'utiliser. Par conséquent les données ne permettent pas de réfuter l'hypothèse $\mathbf{H 2}_{\mathbf{a}^{+}}$(l'utilité perçue a une influence positive et significative sur l'intention d'utilisation). En étudiant la relation entre l'influence sociale et l'intention d'utiliser uniquement, nous avons découvert que la connexion reliant ces deux construits était positive et significative. Cependant, l'effet n'a pas été maintenu dans tous les modèles examinés, même s'il est resté significatif avec l'inclusion de l'utilité perçue et de la commodité. Toutefois, lorsque toutes les variables examinées ici ont été incluses dans le modèle, l'effet de l'influence sociale a complètement disparu. Ainsi l'hypothèse $\mathbf{H} \mathbf{2}_{\mathbf{b}}{ }^{+}$est réfutée.

Les variables extra-fonctionnelles influencent l'intention d'utilisation en particulier l'utilité perçue qui a un effet positif et significatif sur l'intention d'utiliser les services du mobile money. Alors l'hypothèse 2 est validée.

Dans un second temps, il sera question de voir si les conditions facilitantes (commodité, coût perçu) et l'intention d'utilisation influencent l'adoption des innovations de service (AMM). Les résultats obtenus par analyse de régression logistique sont présentés dans le tableau ci-dessous :

Tableau n ${ }^{\circ} 6$ : Résultats de régression logistique binaire

\begin{tabular}{|c|c|c|c|c|c|c|c|}
\hline \multirow{2}{*}{$\begin{array}{c}\mathbf{R}^{2} \\
\text { Nagelkerke }\end{array}$} & \multicolumn{7}{|c|}{ Variables dans l'équation } \\
\hline & & B (béta) & S.E. & Wald & Ddl & (P-value) & $\operatorname{Exp}(\mathbf{B})$ \\
\hline \multirow{5}{*}{0,745} & $\begin{array}{l}\text { FACT_COM } \\
(C M A \overline{1})\end{array}$ & 1,216 & ,372 & 10,667 & 1 & 0,001 & 3,374 \\
\hline & FACT_CT (CTA1) & $-0,560$ & 0,615 & 0,831 & 1 & 0,362 & 0,571 \\
\hline & FACT_IT (ITAI) & 1,379 & 0,390 & 12,470 & 1 & 0,000 & 3,971 \\
\hline & (Constante) & 5,655 & 1,009 & 31,387 & 1 & 0,000 & 0,000 \\
\hline & \multicolumn{3}{|c|}{ Équation de la régression logistique } & \multicolumn{4}{|c|}{$A M M=1,216 C M A 1-0,560 C T A 1+1,379$ ITA1+5,655 } \\
\hline
\end{tabular}


Il ressort de cette analyse que le coefficient de régression de l'intention d'utilisation $(B=1,379)$ est positivement et statistiquement significatif (P-value < 0,05). En effet, un point additionnel à la variable «intention d'utilisation » multiplierait par 3,971 les chances du consommateur à adopter les services d'argent mobile. Ceci dit, avec les données collectées, l'hypothèse $\mathbf{H 4}^{+}$(l'intention d'utilisation à une influence positive directe et significative sur l'adoption des services) est non réfutée. De même, le coefficient de régression de la commodité $(B=1,216)$ est positivement et statistiquement significatif mais en augmentant d'un $(01)$ point cette variable, cela multiplierait par 3,374 les chances de l'abonné à accepter et à utiliser les services d'argent mobile. Et donc l'hypothèse $\mathbf{H 3}_{\mathbf{a}}{ }^{+}$est non réfutée. Par contre le coefficient de régression du coût perçu $(B=-$ $0,560)$ est négatif et non significatif $(0,362>0,05)$. Ce qui explique que la variable « coût perçu » n'influence pas négativement l'utilisation complète. Dit autrement, que l'adoption des services d'argent mobile par les consommateurs dépend en partie de son coût abordable. Par conséquent l'hypothèse $\mathbf{H 3}_{\mathbf{b}}{ }^{-}$est réfutée. Les conditions facilitantes influencent l'adoption des services notamment la commodité qui a une forte influence sur l'adoption et donc l'hypothèse 3 est validée. Toutefois, il faut souligner l'existence d'un pouvoir explicatif qui demeure fort $\left(\mathrm{R}^{2}\right.$ Nagelkerke $\left.=74,5 \%>50 \%\right)$ des variables mobilisées à savoir : la commodité et l'intention comportementale.

\section{B. LA DISCUSSION DES RESULTATS DE LA RECHERCHE}

Les méthodes d'équations structurelles sont habituellement mobilisées dans la plupart des travaux portant sur l'adoption de la banque mobile, du commerce électronique et du paiement mobile. Dans ce papier les relations entre les construits de l'UTAUT, avec l'ajout de la testabilité et du risque perçu, ont été examinées à l'aide d'une analyse de régression linéaire et logistique, pour comprendre l'intention comportementale et l'adoption des services du mobile money (Carlsson et al., 2006 ; Bellahcene et Khedim, 2016 ; Fall et al., 2019).

\section{Implications théoriques}

L'acceptation et l'utilisation du système d'informations dans un contexte organisationnel montre que la performance espérée (utilité perçue) constitue le principal moteur de l'intention comportementale des employés en matière d'utilisation de la technologie (Venkatesh et al., 2003, 2012). Théoriquement nos résultats suggèrent, dans un contexte non organisationnel, que la variable testabilité représente un facteur essentiel de l'intention comportementale et s'est avérée être un moteur plus important que l'utilité perçue. De même, cette variable testabilité est l'une des caractéristiques intrinsèques de l'innovation (type Rogers) qui vient renchérir les variables de UTAUT dans le contexte sénégalais. Respectivement les deux variables (testabilité et utilité perçue) influencent positivement l'intention d'utiliser le service d'argent mobile (Venkatesh et al, 2003 ; Tobbin et Kuwornu, 2011). En outre les clients de la ville de Dakar utilisent les services parce qu'ils le trouvent très utile dans leurs vies quotidiennes, ce qui les aident à faire beaucoup de choses plus facilement et plus rapidement. 
L'absence d'influence significative de «l'effort attendu » sur l'intention d'utiliser les services du mobile money, bien que surprenante a déjà été observé par d'autres études (Al-Gahtani et al., 2007, Kouakou, 2019). Ce résultat vient à l'encontre de nombreux travaux, à l'instar de Venkatesh et al. (2003), mais peut être expliqué en indiquant qu'avec l'augmentation des années d'expériences concernant l'interaction avec l'innovation, la facilité d'utilisation devient moins importante pour prédire les intentions comportementales des consommateurs à Dakar (Al-Gahtani et al., 2007). En ce qui concerne le risque perçu du modèle, cette variable a une influence négative sur l'intention d'utiliser (Martins et al., 2014 ; Baganzi et al., 2017). Alors on pourrait l'expliquer par le fait que la plupart des utilisateurs se préoccupent du système de sécurité mis en place par les fournisseurs des services, du moment où des informations liées aux falsifications et aux arnaques ne cessent de perdurer. Ce résultat est corroboré par une Enquête Nationale sur les Services Financiers effectuée par l'Observatoire de la Qualité des Services Financiers (OQSF) en 2019 qui révèle que l'insécurité des transactions au Sénégal se chiffre à $15,7 \%$.

L'avis général de l'échantillon sur l'adoption des services du mobile money est synthétisé comme suit : «j'en ai entendu parler grâce aux médias ». Ceci dit, $85 \%$ des interviewés ont déclaré avoir entendu parler des services du mobile money par la stratégie de l'inspirateur (neuromarketing). Pour dire que nos répondants ne se préoccupent pas des facteurs environnementaux tels que les opinions des amis, parents et donc ils ne font pas les choses par mimétisme. C'est ce qui expliquerait l'absence d'effet significatif de l'influence sociale sur l'intention d'utilisation (Carlsson et al., 2006), contredit par d'autres travaux en particulier (Kouakou, 2019).

Également nous avons constaté que l'intention d'utilisation et la commodité ont un effet direct et significatif sur l'adoption de l'innovation de service (Sripalawat et al., 2011 ; Yu, 2012; Bhatiasevi, 2016). Théoriquement, nos résultats suggèrent que près de $74,5 \%$ de la variance de l'utilisation actuelle du système est expliquée par ces deux variables citées précédemment. Cela suggère que nos répondants utilisent réellement les services du mobile money parce qu'ils avaient l'intention de l'utiliser. Également que le service, offre plus d'ubiquité par le fait que les clients/utilisateurs peuvent en profiter n'importe quand, n'importe où, sur n'importe quel appareil et procure des gains de temps bref, qu'il améliore la qualité de vie humaine des consommateurs. Concernant le coût financier, il est à noter qu'il n'a aucun effet significatif sur l'adoption de l'innovation (Mohamad et al., 2018). On retient donc que la plupart des consommateurs trouvent que le coût lié à l'utilisation du système est abordable comparativement aux avantages qu'ils procurent.

En résumé, l'extension de l'UTAUT incorpore non seulement les relations d'UTAUT sauf l'impact des modérateurs, mais intègre de nouvelles variables qui étendent l'applicabilité d'UTAUT au contexte du consommateur. Une étude qualitative prenant en compte les réalités du contexte de l'étude a été effectuée pour peaufiner le modèle et un support empirique a été fourni pour tester ce dernier via une enquête en face en face avec un nombre de 251 consommateurs. La variance expliquée, à la fois dans l'intention comportementale (81,5\%) et l'adoption de l'innovation (74,5\%) est substantielle, par rapport à l'UTAUT de base qui expliquerait respectivement $56 \%$ et $40 \%$ de la variance dans l'intention et l'adoption. Cela suggère que les extensions 
SOSSOU et al.: Facteurs explicatifs de l'adoption des services du mobile money par les clients des opérateurs...

proposées sont essentielles pour rendre la validité prédictive de UTAUT dans un contexte individuel à ce qui a été trouvé dans l'étude de l'UTAUT original dans un contexte organisationnel (Venkatesh et al., 2003, 2012). Cette étude propose enfin une extension du modèle UTAUT intégrant la testabilité comme facteur explicatif de l'adoption des services du mobile money dans le contexte sénégalais plus spécifiquement à Dakar.

\section{Implications managériales}

A l'issue des résultats obtenus, il a été constaté que la testabilité est un facteur important affectant l'intention d'utiliser les services du mobile money par les consommateurs. Par conséquent, les gestionnaires doivent donner la possibilité aux clients d'essayer et de tester le service d'argent mobile afin d'avoir une idée sur son fonctionnement (Tobbin et Kuwornu, 2011). Nous constatons empiriquement que le risque perçu affecte négativement et significativement l'intention d'utiliser les services du mobile money. Ceci dit, les gestionnaires doivent avant tout s'assurer qu'une plateforme d'argent mobile est techniquement solide avec de bonnes pratiques de sécurité mises en place pour minimiser les risques des utilisateurs finaux. Par ailleurs, bien vrai que le risque zéro n'existe pas mais les gestionnaires devraient aussi annoncer aux consommateurs que l'utilisation des services du mobile money est sécurisé en véhiculant des informations de sécurité et de confiance du système. Ensuite empêcher aux utilisateurs de se préoccuper, des délits informatifs, des atteintes à la vie privée et des informations liées aux arnaques. Mettre en confiance ses clients en les rassurant que l'utilisation du canal est sécurisée entre leur téléphone portable/smartphone et le serveur des opérateurs (Martins et al., 2014 ; Baganzi et al., 2017). Par ailleurs, inclure des garanties de remboursement et des garanties de satisfaction des consommateurs, afin que ces derniers se sentent à l'aise et en sécurité avec ledit système. Bref, le service doit assurer le respect de la vie privée des consommateurs sur le mobile. Aussi nos résultats suggèrent que le coût perçu (valeur du prix) n'affecte pas l'adoption des services qu'offre l'innovation. Dit autrement, que les avantages perçus du service par rapport au sacrifice monétaire, peuvent influencer les utilisateurs à l'adopter (Venkatesh et al., 2012). Le mobile money est donc destiné aux personnes à faible revenu, ce qui prône l'intégration de toutes les classes sociales. Ceci pourrait permettre à la BCEAO d'accélérer son taux d'inclusion financière en donnant une opportunité aux populations mal desservies par les services financiers classiques. Ce faisant dans une perspective de lutte contre la pauvreté, l'offre des services à faible coût pourrait permettre aux banques et prestataires de toucher une large couche de clientèle les plus pauvres vivant dans les zones reculées (Diniz et al., 2011). Dans la même veine, il faudrait que les autorités monétaires et de régulation accélèrent la mise en œuvre du projet d'interopérabilité qui faciliterait davantage l'exécution des opérations et des services financiers mobiles entre clients appartenant à différents banques et opérateurs techniques. Néanmoins les gestionnaires peuvent mettre en place des stratégies de promotion et de tarification en réduisant surtout le pourcentage des frais de retrait afin de maintenir voire augmenter leur portefeuille clients. Comme observé l'un des répondants affirme et je cite : "le coût lié au retrait est vraiment cher et on ne peut pas supporter de payer les frais à chaque fois que l'on cherche à récupérer nos fonds » 
(A.K, représente les initiales de l'interviewé qui a 28ans et de sexe Féminin). Pour finir les gestionnaires doivent orienter des stratégies de communication commerciale adaptées à chaque cible car les besoins et comportements diffèrent, ce qui pourrait déterminer le comportement de ces derniers lors d'un achat ou face à une publicité. Mais aussi trouver une stratégie avec la BCEAO pour déplafonner le compte électronique qui se chiffre à 2 millions FCFA.

\section{Limites et recherches futures}

Bien que cette étude vienne apporter sa contribution aussi minime soit-elle aux connaissances existantes, nous reconnaissons également ses limites, principalement en ce qui concerne la taille et la méthode d'échantillonnage. Les participants pour la plupart étaient des jeunes très instruits dont le comportement peut différer comparé à la moyenne du grand public. Ils sont généralement des technophiles qui acceptent plus rapidement des innovations technologiques, ce qui peut impacter sur nos conclusions. La probabilité est aussi forte à ce que les consommateurs analphabètes, plus âgés ou des technophobes aient une grande difficulté à utiliser le service d'argent mobile tout en étant confrontés aux risques inhérents plus élevés. Aussi n'étudié qu'un seul type d'innovation (mobile money) et de limiter l'étude à une zone urbaine peuvent être discutés. Les recherches futures peuvent s'appuyer sur cette étude en testant ce modèle dans différentes technologies, différents groupes d'âges, différents pays et dans d'autres contextes. Par ailleurs, les recherches peuvent également intégrer des variables pertinentes qui pourraient mieux expliquer l'intention comportementale d'utiliser les services du mobile money, comme l'habitude et la confiance perçue (variables décelées lors de l'enquête) sans oublier de prendre en compte les variables modératrices (âge, sexe, niveau d'étude, revenu, expérience d'utilisation).

\section{CONCLUSION}

Ce papier est le résultat d'un travail de recherche qui est toujours en cours. Son objectif étant de partager avec les chercheurs les premiers résultats déjà obtenus. Sur le marché sénégalais, les dispositions des services du mobile money qu'offrent les OTM sont similaires. A l'appui de UTAUT avec l'ajout du risque perçu et surtout de la testabilité obtenue dans l'étude qualitative, mobilisé pour prédire les réels facteurs à prendre en compte dans l'adoption des services du mobile money, il ressort dans les conclusions que la régression linéaire montre par ailleurs que la testabilité et l'utilité perçue représentent respectivement les facteurs les plus significatifs de l'intention d'utiliser les services d'argent via le mobile par les clients. Dans le même ordre d'idées la régression logistique montre à son tour que l'intention d'utilisation et la commodité représentent respectivement les facteurs qui influencent positivement et significativement l'adoption de l'innovation incrémentale de service. Cependant l'influence sociale et l'effort attendu n'ont aucune influence significative sur l'intention d'utiliser les services. Enfin le risque perçu représente un facteur qui influence négativement et significativement l'intention d'utiliser alors que le coût perçu n'a aucun effet significatif sur l'adoption des services. Conscient du pouvoir explicatif du modèle de l'UTAUT évolué, la variance expliquée à la fois dans l'intention comportementale $(81,5 \%)$ et l'utilisation de l'innovation $(74,5 \%)$ n'est pas négligeable, ce qui pourrait 
représenter une autre contribution pour enrichir la recherche des facteurs explicatifs de l'adoption des services du mobile money.

\section{BIBLIOGRAPHIE}

[1] Aboelmaged, M., \& Gebba, T. R. Mobile Banking adoption : an examination of technology acceptance model and theory of planned behavior. International Journal of Business Research and Development, 2013, 2(1), pp. $35-50$

[2] Agarwal, R., \& Prasad, J. The role of innovation characteristics and perceived voluntariness in the acceptance of information technologies. Decision sciences, 1997, 28(3), 557-582.

[3] Ajzen, I. The theory of planned behavior. Organizational behavior and human decision processes, 1991, 50(2), 179-211.

[4] Alkhunaizan, A., \& Love, S. What drives mobile commerce? An empirical evaluation of the revised UTAUT model. International Journal of Management and Marketing Academy, 2012, 2(1), 82-99.

[5] Al-Gahtani, S. S., Hubona, G. S., \& Wang, J. Information technology (IT) in Saudi Arabia : Culture and the acceptance and use of IT. Information \& management, 2007,44(8), 681-691.

[6] Al-Jabri, I. M. The intention to use mobile banking: Further evidence from Saudi Arabia. South African Journal of Business Management, 2015, 46(1), 23-34.

[7] Anderson, R. E., \& Srinivasan, S. S. E-satisfaction and e-loyalty: A contingency framework. Psychology et marketing, 2003, 20(2), 123-138.

[8] Baganzi, R., \& Lau, A. Examining trust and risk in mobile money acceptance in Uganda. Sustainability, 2017, 9(12), 2233.

[9] Baptista, G., \& Oliveira, T. A weight and a meta-analysis on mobile banking acceptance research. Computers in Human Behavior, 2016, 63, 480-489.

[10] Basri, S. Determinants of adoption of mobile banking: evidence from rural Karnataka in India. International Journal of Trade and Global Markets, 2018, 11(1-2), 77-86.

[11] Bauer, R. A. Consumer behavior and risk taking in risk taking and information handling in consumer behavior, Edited by: Donald F. Cox, 1967.

[12] Berry, L. L., Seiders, K., \& Grewal, D. Understanding service convenience. Journal of marketing, 2002, 66(3), 1-17.

[13] Bhatiasevi, V. An extended UTAUT model to explain the adoption of mobile banking. Information Development, 2016, 32(4), 799-814.

[14] Brown, L.G. Convenience in services marketing”, Journal of Services Marketing, 1990, Vol. 4, Winter, pp. 53-9.

[15] Buabeng-Andoh, C. Factors influencing teachersâ adoption and integration of information and communication technology into teaching: A review of the literature. International Journal of Education and Development using ICT, 2012, 8(1), pp. 136-155. 
[16] Chaix, L., \& Torre, D. Le double rôle du paiement mobile dans les pays en développement. Revue économique, 2015, 66(4), 703-727.

[17] Chauhan, S. Acceptance of mobile money by poor citizens of India: Integrating trust into the technology acceptance model. Info, 2015, 17(3), 58-68.

[18] Chen, H. Individual Mobile Communication Services and Tariffs (No. EPS-2008-123-LIS). 2008.

[19] Cheikho, A. L'adoption des innovations technologiques par les clients et son impact sur la relation client-Cas de la banque mobile (Doctoral dissertation). 2015.

[20] Choudrie, J., Junior, C. O., McKenna, B., \& Richter, S. Understanding and conceptualising the adoption, use and diffusion of mobile banking in older adults: A research agenda and conceptual framework. Journal of Business Research, 2018, 88, 449-465.

[21] De Bonville, J., \& Guerard, G. L'analyse de contenu des medias: de la probleme au traitement statistique. Canadian Journal of Communication, 2000, 25(4), 568.

[22] Evrard, Y., Pras, B., Roux, E., Desmet, P., Dussaix, A. M., \& Lilien, G. L. Market-Fondements et méthodes des recherches en marketing (No. hal-00490724).2009.

[23] Fall, F. \& Birba, O. (2019). L'inclusion financière par le mobile-banking au Sénégal: l'analyse des facteurs socio-économiques d'adoption. Mondes en développement, 2019, 185(1), 61-82.

[24] Featherman, M.S. et Pavlou, P.A. Predicting e-services adoption: a perceived risk facets perspective. International Journal of Human-Computer Studies, 2003, Vol59, nº 4, p. 451-474.

[25] Fishbein, M. \& Ajzen, I. Belief, attitude, intention and behavior: an introduction to theory and research, Reading. MA, Addison Wesley, 1975, vol. 10(2)

[26] Hsieh, H. L., Kuo, Y. M., Wang, S. R., Chuang, B. K., \& Tsai, C. H. A study of personal health record user's behavioral model based on the PMT and UTAUT integrative perspective. International journal of environmental research and public health, 2017, 14(1), 8.

[27] Kalakota, R., \& Whinston, A. B. Electronic commerce: a manager's guide. Addison-Wesley Professional.1997.

[28] Jolibert, A., \& Gavard-Perret, M. L. Méthodologie de la recherche en sciences de gestion: réussir son mémoire ou sa thèse en sciences de gestion. Pearson, $2^{\mathrm{e}}$ édition.2012.

[29] Khatimah, H., Susanto, P., \& Abdullah, N. L. Hedonic Motivation and Social Influence on Behavioral Intention of E-Money : The Role of Payment Habit as a Mediator. International Journal of Entrepreneurship, 2019, 23(1), 1-9.

[30] Kikulwe, E. M., Fischer, E., \& Qaim, M. Mobile money, smallholder farmers, and household welfare in Kenya. PloS one, 2014, 9(10): e109804.

[31] Kim, M. J., Chung, N., \& Lee, C. K. (2011). The effect of perceived trust on electronic commerce : Shopping online for tourism products and services in South Korea. Tourism Management, 2011, 32(2), 256-265.

[32] Kouakou, K. S. Les déterminants de l'adoption de l'apprentissage mobile par les étudiantes de l'université Cheikh Anta Diop de Dakar Determinants affecting acceptance level of mobile learning among schoolgirls of the Cheikh Anta Diop university of Dakar, 2019, (15), pp.17-32. 
SOSSOU et al.: Facteurs explicatifs de l'adoption des services du mobile money par les clients des opérateurs...

[33] Lafraxo, Y., Hadri, F., Amhal, H., \& Rossafi, A. The Effect of Trust, Perceived Risk and Security on the Adoption of Mobile Banking in Morocco. In ICEIS, 2018, (2) (pp. 497-502).

[34] Lapointe, J.J. La méthodologie des systèmes souples appliquée à l'amélioration de situations problématiques complexes en éducation - Notes de cours. Département de didactique, de psychopédagogie et de technologie éducative, Faculté des sciences de l'éducation, Université Laval, Ste-Foy, Québec.1996.

[35] Lichtenstein, S., \& Williamson, K. Understanding consumer adoption of internet banking: an interpretive study in the Australian banking context. Journal of electronic commerce research, 2006, 7(2), 50.

[36] Limayem, M., \& Rowe, F. Comparaison des facteurs influençant les intentions d'achat à partir du Web à Hong Kong et en France: Influence sociale, risques et aversion pour la perte de contact. Revue française du marketing, 2006, 209(4/5), 25-48.

[37] Lu, G., Cheng, B., Lin, Q., \&Wang, Y. Quantitative indicator of homeostatic risk perception in car following. Safety science, 2012, 50(9), 1898-1905.

[38] Lu, Y., Cao, Y., Wang, B., \&Yang, S. A study on factors that affect users' behavioral intention to transfer usage from the offline to the online channel. Computers in Human Behavior, 2011, 27(1), 355-364.

[39] Luarn, P., \& Lin, H. H. A customer loyalty model for e-service context. J. Electron. Commerce Res., 2003, 4(4), 156-167.

[40] Lubua, E. W., \& Semlambo, A. The influence of the ease of use and perceived usefulness to the adoption of mobile money services in SMEs in Tanzania. Information Technologist (The), 2017, 14(2), 131-141.

[41] Martins, C., Oliveira, T., \& Popovič, A. Understanding the Internet banking adoption : A unified theory of acceptance and use of technology and perceived risk application. International Journal of Information Management, 2014, 34(1), 1-13.

[42] Maurer, B. (2008). Retail electronic payments systems for value transfers in the developing world. Department of Anthropology, University of California Irvine :

[43] Mensah, I. K., Chuanyong, L., \& Zeng, G. Factors determining the continued intention to use mobile money transfer services (MMTS) among university students in Ghana. International Journal of Mobile Human Computer Interaction (IJMHCI), 2020, 12(1), 1-21

[44] Min, Q., Ji, S., \& Qu, G. Mobile commerce user acceptance study in China : a revised UTAUT model. Tsinghua Science and Technology, 2008,13(3), 257-264.

[45] Mlaiki, A., Kefi, H., \& Kalika, M. Facteurs psychosociaux et continuité d'utilisation des réseaux sociaux numériques : le cas de facebook. Recherches en Sciences de Gestion, 2012, (5), 83-111.

[46] Mohamad, S. A., \& Kassim, S. Examining the Relationship between UTAUT Construct, Technology Awareness, Financial Cost and E-Payment Adoption among Microfinance Clients in Malaysia. In 1st Aceh Global Conference, 2019, (pp. 351-357). Atlantis Press.

[47] Nasri, W. Factors influencing the adoption of internet banking in Tunisia. International journal of business and management, 2011, 6(8), 143-160.

[48] Nel, J., \& Boshoff, C. Enhancing the use of internet banking in an emerging market. South African journal of economic and management sciences, 2014, 17(5), 624-638. 
[49] Njoroge, P. Financial Inclusion in Sub-Saharan Africa; Central Bankers' Speeches; Central Bank of Kenya: Nairobi, Kenya.2016.

[50] Nunnally, J.C. Psychometric Theory. New York, NY: McGraw-Hill.1978.

[51] Polatoglu, V. N., \& Ekin, S. An empirical investigation of the Turkish consumers' acceptance of Internet banking services. International journal of bank marketing, 2011, 19(4),156-165

[52] Ozdemir, S., \& Trott, P. Exploring the adoption of a service innovation: A study of Internet banking adopters and non-adopters. Journal of Financial Services Marketing, 2009,13(4), 284-299.

[53] Upadhyay, P., \& Jahanyan, S. Analyzing user perspective on the factors affecting use intention of mobile based transfer payment. Internet Research,2016, 26, 38-56.

[54] Roca, J. C., García, J. J., \& De La Vega, J. J. The importance of perceived trust, security and privacy in online trading systems. Information Management \& Computer Security,2009,17(2), 96-113.

[55] Rogers, E. M. Diffusion of Innovations: modifications of a model for telecommunications. In Die diffusion von innovationen in der telekommunikation, 1995, (pp. 25-38). Springer, Berlin, Heidelberg.

[56] Schierholz, R., \& Laukkanen, T. Internet vs mobile banking: Comparing customer value perceptions. Business process management journal, 2007, 13, 788-797

[57] Shaikh, A. A., \& Karjaluoto, H. Mobile banking adoption : A literature review. Telematics and informatics, 2015,32(1), 129-142.

[58] Siddik, M. N. A., Sun, G., Yanjuan, C. U. I., \& Kabiraj, S. Financial inclusion through mobile banking: a case of Bangladesh. Journal of Applied finance and Banking,2014, 4(6), 109.

[59] Slade, E. L., Dwivedi, Y. K., Piercy, N. C., \& Williams, M. D. Modeling consumers' adoption intentions of remote mobile payments in the United Kingdom: extending UTAUT with innovativeness, risk, and trust. Psychology \& Marketing, 2015, 32(8), 860-873.

[60] Stewart, K. J. Trust transfer on the world wide web. Organization Science, 2003, 14(1), 5-17.

[61] Tan, G. W. H., Ooi, K. B., Chong, S. C., \& Hew, T. S. NFC mobile credit card: the next frontier of mobile payment?. Telematics and Informatics, 2014, 31(2), 292-307.

[62] Thietart \& al. (2014). Méthodes de recherche en management. DUNOD, $4^{\mathrm{e}}$ édition

[63] Tobbin, P., \& Kuwornu, J. K. Adoption of mobile money transfer technology: structural equation modeling approach. European Journal of Business and Management, 2011, 3(7), 59-77.

[64] Turban, E., King, D., Lee, J., et Viehland, D. Electronic commerce : A managerial perspective 2002. Prentice Hall : ISBN 0,2002, 13(975285), 4.

[65] Venkatesh, V., Morris, M. G., Davis, G. B., et Davis, F. D. User acceptance of information technology : Toward a unified view. MIS quarterly, 2003, 425-478.

[66] Venkatesh, V, Thong, J. YL, et Xu, X. Consumer acceptance and use of information technology: extending the unified theory of acceptance and use of technology. MIS quarterly, 2012, 36(1), 157-178.

[67] Yang, S., Lu, Y., Gupta, S., Cao, Y., \& Zhang, R. Mobile payment services adoption across time : An empirical study of the effects of behavioral beliefs, social influences, and personal traits. Computers in Human Behavior,2012, 28(1), 129-142. 
SOSSOU et al.: Facteurs explicatifs de l'adoption des services du mobile money par les clients des opérateurs...

[68] Yousafzai, S. Y., Pallister, J. G., \& Foxall, G. R. A proposed model of e-trust for electronic banking. Technovation,2003, 23(11), 847-860.

[69] Yousafzai, S., Pallister, J., \& Foxall, G. Multi-dimensional role of trust in Internet banking adoption. The Service Industries Journal,2009, 29(5), 591-605.

[70] Yu, C. S. Factors affecting individuals to adopt mobile banking: Empirical evidence from the UTAUT model. Journal of electronic commerce research,2012, 13(2), 104.

Annexe : A

\begin{tabular}{|c|c|c|}
\hline Unité de sens & Unité d'enregistrement & Occurrences \\
\hline $\begin{array}{c}\text { Facilité } \\
\text { d'utilisation }\end{array}$ & $\begin{array}{l}\text { - Pas difficile à utiliser les services d'argent mobile. } \\
\text { - L'utilisation du service est rendu facile par le téléphone. } \\
\text { - Je suis très satisfait des services de mobile money à cause de la } \\
\text { facilité des transactions. Les étapes pour les différentes transactions } \\
\text { sont intuitives, interactives et simples } \\
\text { Total }\end{array}$ & $\begin{array}{l}5 \\
1 \\
1 \\
1 \\
\mathbf{8}\end{array}$ \\
\hline $\begin{array}{c}\text { Commodité du } \\
\text { service }\end{array}$ & $\begin{array}{l}\text { - Le service d'argent mobile procure des gains de temps, accessible, } \\
\text { pratique et nécessite peu d'efforts physiques et mentaux. Il est } \\
\text { innovant (en termes de technologie liée au service financier), } \\
\text { accessible à tous, pratique et disponible } \\
\text { - L'on peut régler ses factures sans se déplacer } \\
\text { - La disponibilité des agents rend plus accessible les dépôts } \\
\text { - Le service d'argent mobile est compatible avec le style de vie de la } \\
\text { population } \\
\text { Total }\end{array}$ & $\begin{array}{l}5 \\
8 \\
4 \\
28 \\
\end{array}$ \\
\hline Utilité perçue & $\begin{array}{l}\text { - Le service d'argent mobile répond à mes besoins et à mes attentes } \\
\text { tout en me procurant des avantages relatifs } \\
\text { - Je crois que l'utilisation des services du mobile money m'aiderait } \\
\text { à être plus performant dans mes activités respectives est j'ai juste } \\
\text { besoin de me déplacer chez le boutiquier du quartier et les } \\
\text { transactions sont rapides } \\
\text {-Aussi c'est un portefeuille électronique qui me permet de moins } \\
\text { circuler avec de la liquidité, ce qui m'évite des agressions parfois } \\
\text { Total }\end{array}$ & 7 \\
\hline Coût du service & $\begin{array}{l}\text { - Le coût moindre du service d'argent mobile offert et on peut payer } \\
\text { ses factures tout en restant chez soi à zéro frais } \\
\text {-Revoir le coût des services à la baisse. } \\
\text { Total } \\
\end{array}$ & $\begin{array}{l}2 \\
5 \\
7\end{array}$ \\
\hline $\begin{array}{c}\text { Influence } \\
\text { sociale }\end{array}$ & $\begin{array}{l}\text {-J'ai connu et adopté le service à cause de mes proches (amis, } \\
\text { parents, pairs). Le battage publicitaire autour du service m'a emmené } \\
\text { à l'utiliser. } \\
\text { Total }\end{array}$ & 14 \\
\hline $\begin{array}{c}\text { Testabilité du } \\
\text { service }\end{array}$ & $\begin{array}{l}\text { - La phase de testabilité est primordiale pour l'adoption de tout } \\
\text { produit/service car connaitre son fonctionnement peut entrainer son } \\
\text { utilisation régulière. Le service m'a semblé plus pratique après } \\
\text { l'avoir testé } \\
\text { - le mobile money reste une innovation et perturbe forcément les } \\
\text { habitudes et donc tout service ou produit aura une phase de test avant } \\
\text { d'être adopté } \\
\text { Total }\end{array}$ & 9 \\
\hline
\end{tabular}

